\title{
Inversion of data containing information on the aerosol particle size distribution using multiple instruments
}

\author{
M. Fiebig*, , C. Stein ${ }^{2}$, F. Schröder ${ }^{3}$, P. Feldpausch, A. Petzold \\ Deutsches Zentrum für Luft- und Raumfahrt Oberpfaffenhofen, Institut für Physik der Atmosphäre, \\ D-82234 Wessling, Germany
}

Received 30 July 2004; received in revised form 26 January 2005; accepted 26 January 2005

\begin{abstract}
An algorithm for inversion of data containing information on particle size distributions is presented that is designed to be true to the input data, does not need an initial guess, does not assume a shape of the size distribution function, yields the smoothest non-negative result consistent with the input data, calculates the uncertainty of the result based on the uncertainty of the input data, and is capable of combining data from more than one instrument type into one inversion result. To test the algorithm, synthetic data of aircraft payloads sensitive for particle diameters $D_{\mathrm{p}}<0.2 \mu \mathrm{m}$ and combining a cascade of condensation particle counters (CPSA) with a differential mobility analyser (DMA), a passive cavity aerosol spectrometer probe (PCASP), and a parallel diffusion battery (PDB) are generated. The CPSA/DMA instrument combination retrieves the log-normal parameters of the Aitken-mode $\left(0.01 \mu \mathrm{m}<D_{\mathrm{p}}<0.2 \mu \mathrm{m}\right)$ with $<1 \%$ uncertainty while the CPSA/PDB combination reaches $\sim 4 \%$ and the CPSA/PCASP combination $\sim 8 \%$ uncertainty. Compared with the CPSA/DMA set-up, the CPSA/PDB and CPSA/PCASP combinations have advantages with respect to temporal resolution and space/weight demands,
\end{abstract}

\footnotetext{
* Corresponding author. National Oceanic and Atmospheric Administration, Climate Monitoring and Diagnostics Laboratory, 325 Broadway, Boulder, CO 80305, USA, Tel.: +1 303497 4647; fax: +1 3034975590.

E-mail addresses: Markus.Fiebig@dlr.de (M. Fiebig), clste@nerc.ac.uk (C. Stein), Franz.Schroeder@mksinst.com (F. Schröder), Philipp.Feldpausch@dlr.de (P. Feldpausch), Andreas.Petzold@dlr.de (A. Petzold).

${ }^{1}$ Present address: NOAA, Climate Monitoring and Diagnostics Laboratory, 325 Broadway, Boulder, CO 80305, USA.

${ }^{2}$ Present address: Natural Environment Research Council, Polaris House, North Star Avenue, Swindon SN2 1EU, United Kingdom.

${ }^{3}$ Present address: MKS Instruments Deutschland GmbH, Schatzbogen 43, D-81829 München, Germany.
} 
respectively. The algorithm is extendable to other instrument types and may also serve as a tool for optimising instruments or instrument packages with respect to specific scientific questions.

(c) 2005 Elsevier Ltd. All rights reserved.

Keywords: Size distribution inversion; Nucleation mode; Size distribution measurement

\section{Introduction}

Since no instrument for measuring the particle size distribution of an aerosol does so directly, measuring this property usually implies an inversion of data containing the information on the size distribution. Numerous approaches have been proposed to solve this problem (see Kandlikar \& Ramachandran, 1999; Lekhtmakher \& Shapiro, 2000 for a review). Ideally, an inversion algorithm should extract the size distribution information from the data while meeting the following objectives:

- Since different measurement techniques are required for obtaining information on the particle size distribution over an extended size range, an algorithm should be capable of combining data from different instrument types.

- The algorithm should make no assumptions on the shape of the size distribution.

- Applying an algorithm that uses an initial guess requires showing that the result is independent of the initial guess. Ideally, an algorithm does not need an initial guess.

- The algorithm should make no trade-off between the fidelity of the inversion result to the input data and the capability of removing structures from the result which are not contained in the data ("smoothness").

- Particle size distributions often cover a concentration range of 4 or more orders of magnitude. An inversion algorithm should exhibit no bias over the whole concentration range.

- Based on the uncertainty of the input data, the algorithm should yield the uncertainty of the inversion result.

- The algorithm should remain stable when the uncertainty of the input data increases.

The algorithm introduced here is designed to meet all these requirements simultaneously. To this end, the inversion problem is separated into two parts, one ensuring the solution's fidelity to the input data, and one for finding the smoothest, non-negative solution in agreement with the input data. Both problems can be solved with established numerical methods. After describing the algorithm, it is tested on synthetical data generated from pre-defined particle size distributions. The instrument combinations assumed for these tests have been used on research aircraft for measuring the particle size distributions in the tropospheric column. Finally, the algorithm is applied to data from instrument combinations simultaneously measuring the particle size distribution of a laboratory test aerosol.

\section{Inversion algorithm}

\subsection{Description of the problem}

When extracting information on the particle size distribution from data obtained by a given instrument, the relevant property describing the instrument is its transfer function. The transfer function $f\left(D_{\mathrm{p}}\right)$ states 
the fraction of particles with diameter $D_{\mathrm{p}}$ that are registered by the instrument. If a set of $m$ instruments, each with a transfer function $f_{i}\left(D_{\mathrm{p}}\right)$, is considered that is sampling an aerosol with a size distribution function $\mathrm{d} N / \mathrm{d} \log D_{\mathrm{p}}$, the measured particle concentration or response $b_{i}$ of each instrument is given by

$$
\int f_{i}\left(D_{\mathrm{p}}^{\prime}\right) \frac{\mathrm{d} N\left(D_{\mathrm{p}}^{\prime}\right)}{\mathrm{d} \log D_{\mathrm{p}}^{\prime}} \mathrm{d} \log D_{\mathrm{p}}^{\prime}=b_{i} .
$$

This results in a set of $m$, so-called Fredholm-equations of the first kind. Since the $f_{i}\left(D_{\mathrm{p}}\right)$ and $b_{i}$ are known, the inversion problem amounts to solving this set of equations for $\mathrm{d} N / \mathrm{d} \log D_{\mathrm{p}}$. Eq. (1) underlines that the measurement process is equivalent to an expansion of $\mathrm{d} N / \mathrm{d} \log D_{\mathrm{p}}$ in the transfer functions of the involved instruments. The $f_{i}\left(D_{\mathrm{p}}\right)$ span the sub-space of those particle size distributions in size distribution space that can be represented by a given set of instruments, each instrument adding one base vector. The part of a particle size distribution that is mapped into the corresponding nullspace cannot be retrieved by any algorithm.

The first step to solving equation system (1) is to transform it into a single matrix equation:

$$
\mathbf{A} \vec{x}=\vec{b}
$$

This can be achieved by discretising the integrals in particle diameter $D_{\mathrm{p}}$. In this case, the vector $\vec{x}$ contains the discretised particle size distribution function $\mathrm{d} N / \mathrm{d} \log D_{\mathrm{p}}$, whereas $\vec{b}$ contains the particle concentrations or responses measured by each instrument. The $m$ rows of matrix A consist of the discretised transfer functions, corresponding to the instrument responses in $\vec{b}$, where the discretisation in particle diameter is the same as for $\vec{x}$. This approach assumes no specific shape of the particle size distribution function. The smallest structure in the size distribution that can be resolved depends only on the resolution of the discretisation and the information content of the data. In order to avoid boundary effects, the discretisation range has to extend well beyond the range in $D_{\mathrm{p}}$ in which the instruments contain information on $\mathrm{d} N / \mathrm{d} \log D_{\mathrm{p}}$. Although all the transfer functions are supposed to be linearly independent, they are often close to linear dependence. The resolution of the discretisation has to be high enough to represent the linear independence of the transfer functions. Depending on the size of the diameter range to be covered, linear and logarithmic spacing are common choices for the spacing of the discretisation.

An alternative way of making the transition from Eq. (1) to Eq. (2) is to expand the transfer functions $f_{i}\left(D_{\mathrm{p}}\right)$ and the particle size distribution function $\mathrm{d} N / \mathrm{d} \log D_{\mathrm{p}}$ in a set of base functions. In this case, $\mathbf{A}$ and $\vec{x}$ would contain the respective expansion coefficients. In this approach, the type of size distribution function that can be represented does not only depend on the information content of the data, but also on the choice of base functions. Intending to reduce the number of assumptions in the inversion process, this alternative is not considered further.

The resulting matrix equation (2) is usually under-determined and ill-posed. Under-determined means that the matrix A contains more columns than rows, which is equivalent to less instrument responses than points in the discretised size distribution function. Ill-posed means that the column vectors of $\mathbf{A}$ are close to linear dependence, which causes the inversion result to be sensitive to uncertainties in the instrument responses.

To select one of the possible solutions of the under-determined problem and to make the problem less sensitive to uncertainties in the instrument data, information in the form of boundary conditions has to be added. One requirement is based on the assumption that the size distribution $\vec{x}$ is as smooth as possible, i.e. it's second derivative is minimal. This assumption is justified by the tendency of microphysical 
processes among the aerosol particles to even out sudden concentration changes with particle size. The other requirement is that every component of $\vec{x}$ is a concentration and therefore has to be non-negative.

\subsection{Description of the algorithm}

In order to solve Eq. (2), the size distribution vector $\vec{x}$ is separated into a vector $\vec{x}_{r}$ and a vector $\vec{x}_{s}$ with $\vec{x}=\vec{x}_{r}+\vec{x}_{s}$. The vector $\vec{x}_{r}$ is an element of the rank of $\mathbf{A}$ and assures that the instrument response of $\vec{x}$ agrees with a given data set $\vec{b}$. The vector $\vec{x}_{s}$ is an element of the nullspace of $\mathbf{A}$ and therefore does not change the instrument response of $\vec{x}$. It is chosen to assure that $\vec{x}$ is both as smooth as possible and non-negative.

In the first step of the algorithm, $\vec{x}_{r}$ is calculated using Singular Value Decomposition (SVD, Press, Teukolsky, \& Vetterling, 1992). The SVD algorithm separates matrix A into three component matrices: $\mathbf{A}=\mathbf{U W V}^{\mathrm{T}}$. If $\mathbf{A}$ has $m$ rows (instruments) and $n$ columns (points in result size distribution), $\mathbf{U}$ has the same dimensions whereas $\mathbf{W}$ and $\mathbf{V}$ are $n \times n$ matrices. The column vectors of $\mathbf{U}$ and $\mathbf{V}$ each form an orthonormal basis, i.e. their transpose equals their inverse. $\mathbf{W}$ is a diagonal matrix. The number of non-zero elements in its diagonal is equal to the rank of A. It can be shown (Press et al., 1992) that the column vectors of $\mathbf{U}$ corresponding to non-zero values in $\mathbf{W}$ form a base of the rank of $\mathbf{A}$. The column vectors of $\mathbf{V}$ corresponding to zero values in $\mathbf{W}$ form a base of the nullspace of $\mathbf{A}$. These properties of $\mathbf{U}$, $\mathbf{W}$, and $\mathbf{V}$ make it easy to calculate the inverse of $\mathbf{A}$ or, if $\mathbf{A}$ is under-determined, its pseudo-inverse:

$$
\vec{x}_{r}=\mathbf{V} \operatorname{diag}\left(\frac{1}{w_{j}}\right) \mathbf{U}^{\mathrm{T}} \vec{b} .
$$

Before the SVD algorithm is applied to matrix $\mathbf{A}$, each line equation of matrix equation (2) is divided by the uncertainty of the respective component of $\vec{b}$. This assures, on the one hand, that each datum is weighted according to its accuracy and, on the other hand, that ranges in the size distribution function with high particle concentrations are not weighted more than size ranges with low particle concentrations.

In the next step, the matrix $\mathbf{V}$ calculated by SVD of $\mathbf{A}$ is used to obtain a base of the nullspace of $\mathbf{A}$. The corresponding matrix $\mathbf{V}_{0}$ contains in its columns the nullspace base vectors. If $k$ is the rank of $\mathbf{A}$, $\mathbf{V}_{0}$ is a $n \times(n-k)$ matrix. Since $\vec{x}_{s}$ is an element of the nullspace of $\mathbf{A}$, it can be expressed as a linear combination of the column vectors of $\mathbf{V}_{0}$ :

$$
\vec{x}_{s}=\mathbf{V}_{0} \vec{a}
$$

where $\vec{a}$ is a $(n-k)$ dimensional vector. With this definition, the criterion that $\vec{x}$ should be smooth and non-negative, i.e. its second derivative should be minimised, can be phrased by the following set of expressions:

$$
\begin{aligned}
& \left\|\mathbf{S}\left(\vec{x}_{r}+\mathbf{V}_{0} \vec{a}\right)\right\| \rightarrow \overrightarrow{0}, \\
& \vec{x}_{r}+\mathbf{V}_{0} \vec{a}>\overrightarrow{0}
\end{aligned}
$$

The matrix $\mathbf{S}$ is the finite-difference representation of the second derivative with the values -2 in its diagonal and 1 in its secondary diagonals and 0 otherwise. The set of expressions in (5) form a linear leastsquares problem with inequality boundary conditions. An algorithm which is capable of solving problems of this kind is the so-called linear least-squares with equality and inequality constraints (LSEI) algorithm 


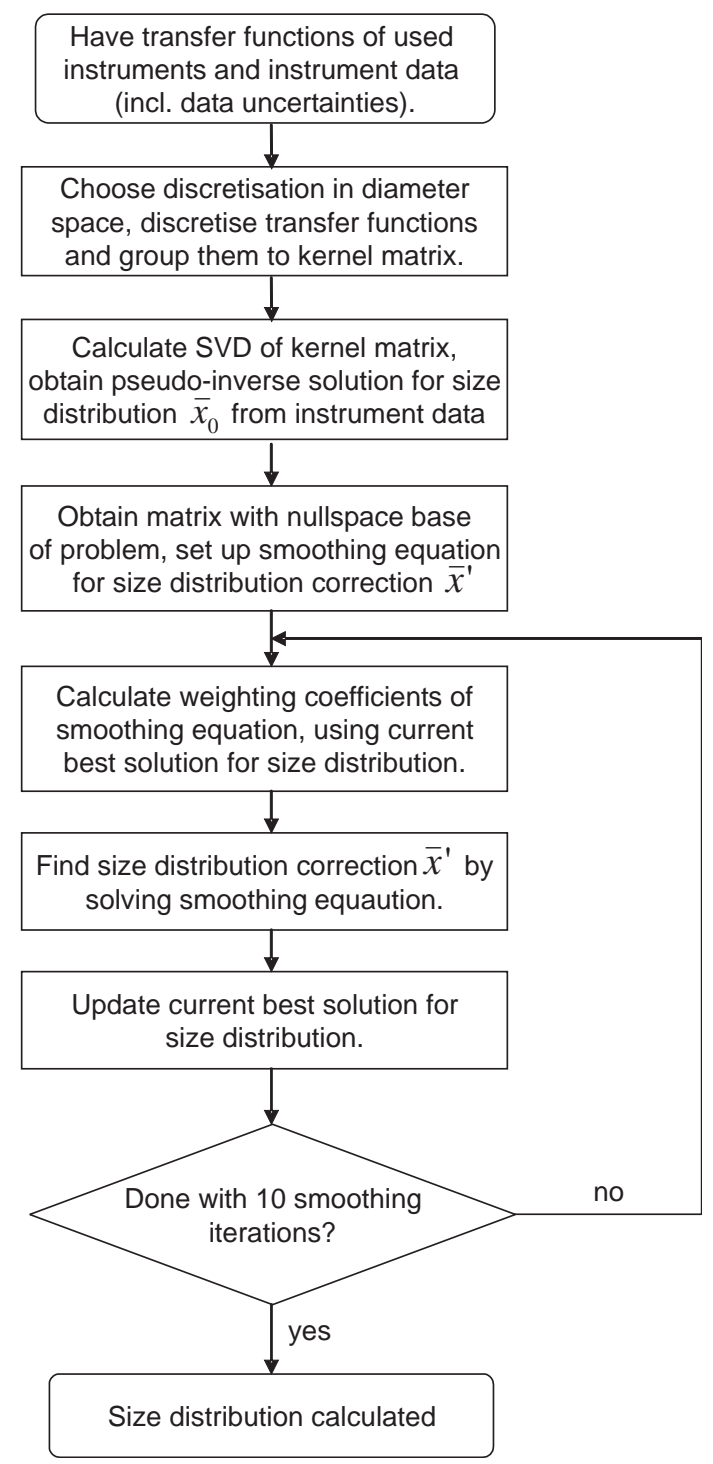

Fig. 1. Flow chart illustrating the steps of the inversion algorithm.

(Haskell \& Hanson, 1981), which is part of the SLATEC numerical library. ${ }^{4}$ This algorithm solves the least-squares problem iteratively by finding in each iteration the vector that yields the strongest improvement of the solution while simultaneously adhering to the constraints when changing the solution vector.

Similar to solving the inversion problem in the rank of $\mathbf{A}$ (Eq. (3)), it has to be made sure that ranges in the particle size distribution with high particle concentrations are not weighted more than ranges with low particle concentrations when solving the inversion problem in the nullspace of $\mathbf{A}$ (Eq. (5)). To this

\footnotetext{
${ }^{4}$ Available at http://www.netlib.org
} 
end, the smoothing matrix $\mathbf{S}$ is substituted by a matrix $\mathbf{S}_{l}^{\prime}$ where each line equation is divided by a power $p_{s}$ of the current concentration in the respective size channel:

$$
\mathbf{S}_{l+1}^{\prime}=\mathbf{S} \operatorname{diag}\left(1 / x_{i, l}^{p_{s}}\right)
$$

To apply Eq. (6), the inversion problem in the nullspace of $\mathbf{A}$ is solved iteratively. The solution of the problem in the range of $\mathbf{A}$ is used to initialise the iteration, $x_{i, 0}=x_{r}$, and to calculate $\mathbf{S}_{1}^{\prime}$. This result is used in Eq. (5) to obtain $\vec{a}$ and, by inserting in $\vec{x}=\vec{x}_{r}+\mathbf{V}_{0} \vec{a}, x_{i, 1}$ which then starts the next iteration cycle. Fig. 1 shows a flow-chart that illustrates the procedure. Usually, 10 iterations are enough to reach convergence.

The described algorithm can be utilised to estimate the uncertainty of the inversion result in two ways. With Error Propagation, the uncertainties of the input data can be used in Eq. (3) to calculate the propagation of these uncertainties to $\vec{x}_{r}$ and thus to $\vec{x}$ (Kandlikar \& Ramachandran, 1999). This method is not strictly accurate in the sense that it assumes the uncertainties of the input data to be normally distributed, which is often not the case. This results in an overestimation of the uncertainty of the inversion result and sometimes in negative values for the lower uncertainty limit of the particle concentration. However, this method is fast and can serve as a first estimate for the uncertainty of the inversion result. In the MonteCarlo method, the input instrument responses in $\vec{b}$ are treated as random variables. Representations of $\vec{b}$ are drawn from this set of random variables and used as input for the inversion algorithm. Each inversion result is stored and the procedure repeated $n$ times ( $n$ typically $>200$ ). The stored inversion results are statistically evaluated to obtain a mean result and its uncertainty. This method is numerically expensive but does not make assumptions on the statistical distribution of the input data. It allows for variables with Gauss, Poisson, or other distributions to be treated. Also, the uncertainty range of the resulting particle size distribution does not reach negative concentrations. Applying a modern algorithm like the LSEI algorithm proves especially useful in combination with the Monte-Carlo method. When the uncertainty of the input data is large, it is possible that combinations of input data values are drawn as random sample that do not allow a non-negative size distribution as inversion result. The LSEI algorithm detects cases which cannot be solved and which therefore have to be rejected. As a result, the Monte-Carlo method only includes inversion results from data samples which are physically meaningful.

\section{Performance tests of algorithm-instrument systems}

Since the transfer functions of a given set of instruments span the sub-space of particle size distribution space representable by the instruments, an assessment of a size distribution inversion algorithm has to be based on the instrument sets and size distributions expected during its use and has to include a discussion of the instruments' transfer functions. Although the range of applications of the introduced algorithm is not limited, only instrument combinations will be considered here that have been deployed onboard the Falcon research aircraft, operated by the Deutsches Zentrum für Luft-und Raumfahrt (DLR), in order to characterise the atmospheric particle size distribution between the planetary boundary layer and the lowermost stratosphere. The instruments considered comprise the condensation particle size analyser (CPSA), i.e. a cascade of four condensation particle counters (CPCs), a differential mobility analyser (DMA), a passive cavity aerosol spectrometer probe 100X (PCASP) optical particle counter, and a parallel diffusion battery (PDB).

These performance tests are conducted by defining a set of test particle size distributions that represent a wide range of aerosols that could be encountered in real measurements, in this case in the troposphere 
Table 1

Parameters of particle size distributions consisting of two log-normal modes (nucleation- and Aitken-mode) used to test the instrument performance

\begin{tabular}{|c|c|c|c|c|c|c|}
\hline \multirow[t]{2}{*}{ Label } & \multicolumn{3}{|c|}{ Nucleation mode } & \multicolumn{3}{|c|}{ Aitken-mode } \\
\hline & $N_{\text {int }}$ & $D_{\text {med }}$ & $\sigma_{\text {geom }}$ & $N_{\text {int }}$ & $D_{\text {med }}$ & $\sigma_{\text {geom }}$ \\
\hline (a) & 40000 & 0.0008 & 1.7 & 400 & 0.03 & 1.7 \\
\hline (b) & 40000 & 0.0008 & 1.7 & 400 & 0.05 & 1.7 \\
\hline (c) & 40000 & 0.0008 & 1.7 & 400 & 0.08 & 1.7 \\
\hline (d) & 40000 & 0.0042 & 1.7 & 400 & 0.03 & 1.7 \\
\hline (e) & 40000 & 0.0042 & 1.7 & 400 & 0.05 & 1.7 \\
\hline (f) & 40000 & 0.0042 & 1.7 & 400 & 0.08 & 1.7 \\
\hline
\end{tabular}

and lowermost stratosphere. For these test size distributions, the responses of the considered instruments are calculated by using Eqs. (1) and (2). These responses, essentially "artificial measurements", are used as input for the inversion algorithm. The inversion result is compared with the initial test particle size distribution. The particle size range of interest here consists of the nucleation mode $\left(D_{\mathrm{p}}<0.01 \mu \mathrm{m}\right)$ and the Aitken-mode $\left(0.01 \mu \mathrm{m}<D_{\mathrm{p}}<0.2 \mu \mathrm{m}\right)$ size ranges. The test particle size distributions, given in Table 1, therefore consist of these two log-normal modes. The range of the three median diameters $D_{\text {med }}$ of the Aitken-mode chosen for the test size distributions covers the range encountered in the free troposphere (Petzold et al., 2002). The integral particle concentration $N_{\text {int }}$ and the geometric standard deviation $\sigma_{\text {geom }}$ chosen for this mode represent typical values in this region of the atmosphere at mid-latitudes. This is a more rigorous test for an inversion algorithm than using a higher particle concentration, e.g. from the boundary layer, since lower concentrations have a larger uncertainty and place a higher demand on the algorithm's stability. Each of the three parameter sets for the Aitken-mode is combined with two different sets of parameters for the nucleation mode which were obtained during the New Particle Formation and Fate in the Coastal Environment (PARFORCE) project (O'Dowd et al., 2002; Pirjola, O'Dowd, \& Kulmala, 2002). In the distributions (a)-(c) (see Table 1), the three different Aitken-mode distributions are paired with a nucleation mode distribution without significant formation of new particles. In contrast, the cases (d)-(f) represent conditions with recent new particle formation.

The agreement of each inversion result $\mathrm{d} N_{\text {inv }}\left(D^{\prime}\right) / \mathrm{d} \log D^{\prime}$ with the true size distribution $\mathrm{d} N_{\text {true }}\left(D^{\prime}\right) /$ $\mathrm{d} \log D^{\prime}$ is quantified by calculating its logarithmic $\chi^{2}$ value:

$$
\chi_{\log }^{2}=\int\left[\log \left(\frac{\mathrm{d} N_{\text {inv }}\left(D^{\prime}\right)}{\mathrm{d} \log D^{\prime}}\right)-\log \left(\frac{\mathrm{d} N_{\text {true }}\left(D^{\prime}\right)}{\mathrm{d} \log D^{\prime}}\right)\right]^{2} \mathrm{~d} \log D^{\prime} .
$$

Also, two-modal logarithmic size distributions are fitted to the inversion results using the LevenbergMarquardt algorithm (Press et al., 1992) and the parameters of the Aitken-mode compared to their true values.

\subsection{Condensation particle size analyser and differential mobility analyser}

The CPSA (Stein, Schröder, \& Petzold, 2001) consists of four CPCs in a configuration similar to the N-MASS instrument by Brock et al. (2000). CPCs count the total number of particles larger than an 


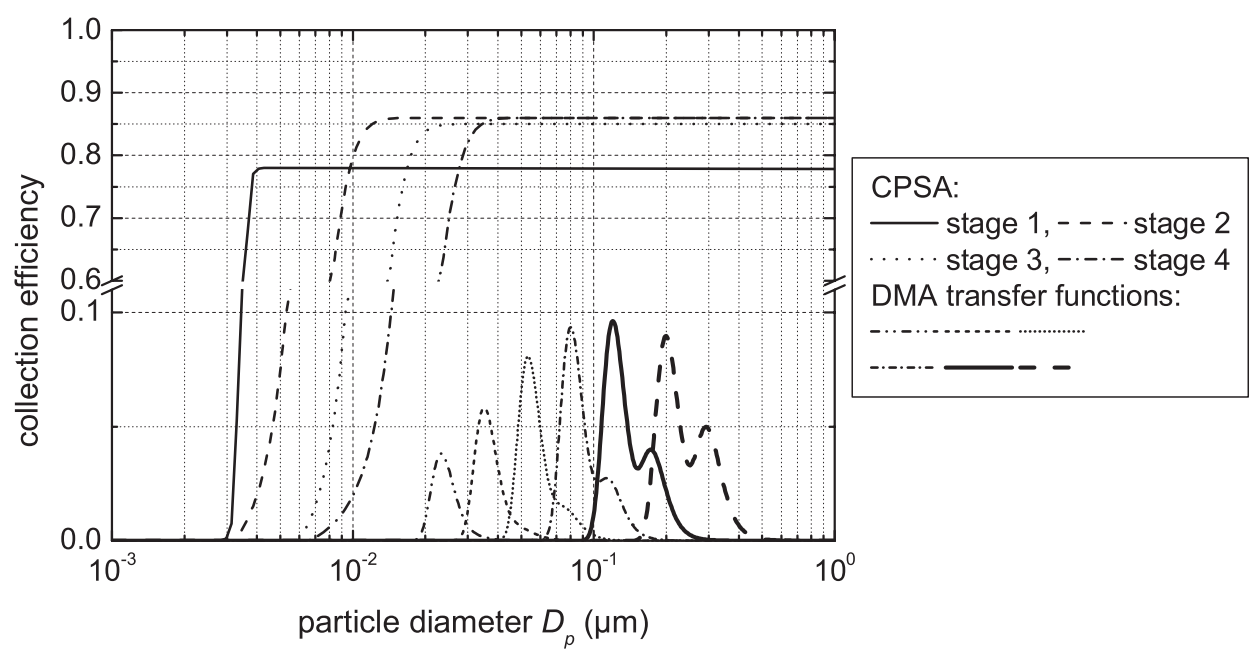

Fig. 2. Transfer functions of the four channels of the CPSA for typical operating conditions, together with transfer functions of the DMA for the voltages selected for this study.

approximate threshold diameter. The particles are activated by exposing them to a supersaturated alcohol atmosphere and registered by counting the light pulses caused by the particles while traversing a laser beam. The activation threshold diameter depends on the supersaturation reached in the instrument. Information on the particle size distribution is obtained by combining several CPCs with differing activation threshold diameters. In the standard configuration, the four CPSA channels reach $50 \%$ of the maximum counting efficiency at 3.5, 6.5, 9.0, and $21 \mathrm{~nm}$ particle diameter, respectively. While a detailed account of the instrument's characteristics and calibration is given in Appendix A, the transfer functions of the CPSA channels under standard operating conditions, which were also used for the performance analysis, are plotted in Fig. 2.

For the first performance test, the CPSA is combined with a DMA. This set-up maximises the size range where information is obtained on the size distribution while avoiding the DMA's statistical uncertainty in the nucleation mode size range. When measuring the particle size distribution with a DMA, the capacitor voltage can be varied in continuous or a stepwise scan. For a continuous scan, the particle concentration at the exit of the DMA capacitor is typically measured with $10 \mathrm{~Hz}$ resolution. As a result, applying the continuous scan method requires a particle concentration high enough to obtain a significant count rate in every $0.1 \mathrm{~s}$ interval. This is not the case when sampling free-tropospheric aerosol on-board an aircraft. The DMA which is used for the performance study is consequently operated in a stepwise scan mode with six steps between $20 \mathrm{~nm}$ and $0.2 \mu \mathrm{m}$ particle diameter. The respective transfer functions (Roth, Berlauer, \& Heyder, 1989; Wiedensohler, 1988) are also plotted in Fig. 2.

Due to the high information content of the DMA data, this performance test also investigates the overall capabilities of the algorithm. Fig. 3 shows the result by comparing the six test size distributions of Table 1 (solid black lines) with the output of the inversion algorithm (black dots connected by line). The agreement between test size distribution and inversion result is almost perfect with an average $\chi_{\text {log }}^{2}$ value of 0.0011 and only slight deviations at sharp local minima. The algorithm shows no significant bias in the quality of the inversion with respect to particle concentration although the particle concentration changes by over 4 orders of magnitude over the considered size range. 


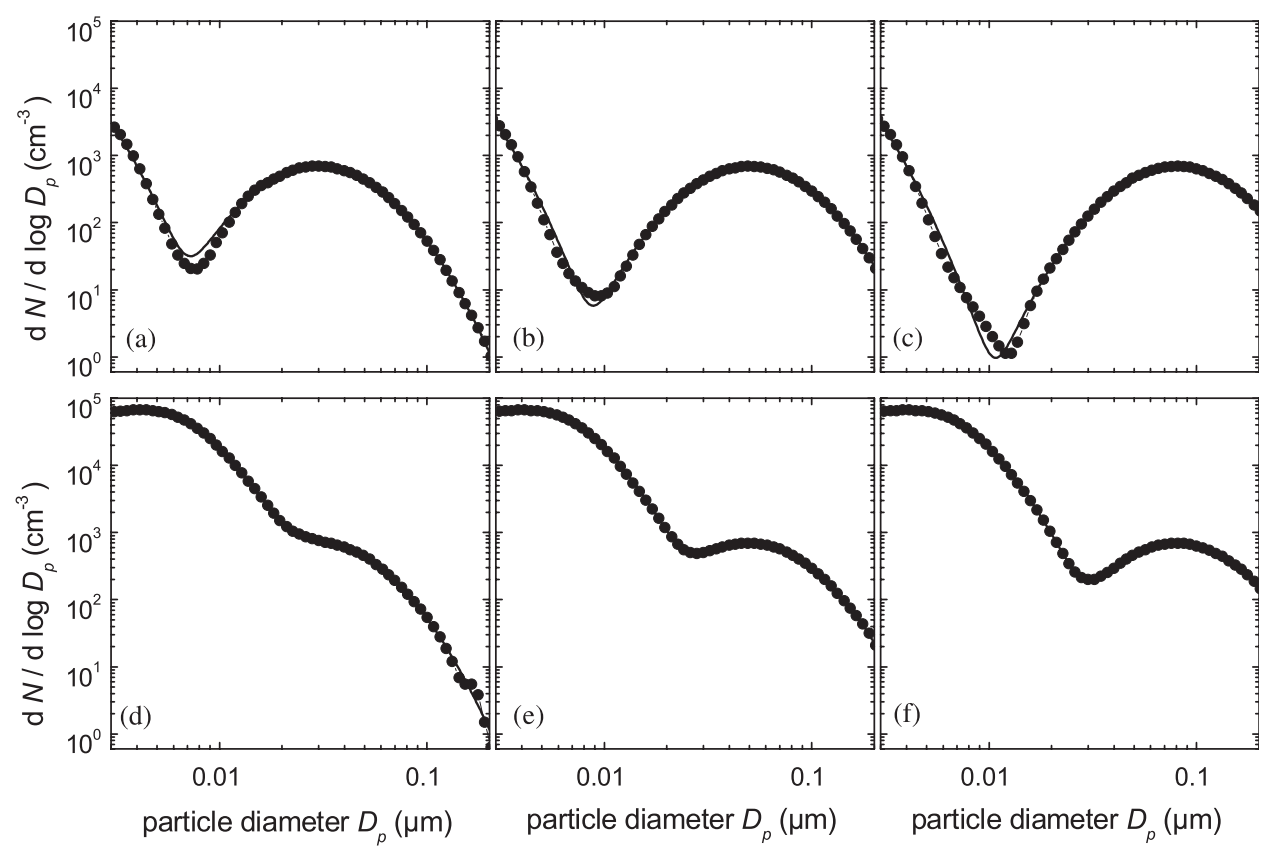

Fig. 3. Comparison of test particle size distributions (Table 1, thin solid lines) and particle size distributions inverted using synthetical instrument responses of CPSA and DMA as input (black dots). The respective ranges in $D_{\mathrm{p}}$ where CPSA and DMA data contain information on the particle size distribution appear in different hatch styles.

However, real data is associated with an uncertainty. For measurements based on the counting of events, the uncertainty is governed by a Poisson distribution. Therefore, the test was repeated while the MonteCarlo method was applied and random samples were drawn from the calculated instrument responses assuming a Poisson distribution. Fig. 4 shows the outcome with the uncertainty range of the inversion result depicted in grey. The agreement between test size distribution and inversion result is still very good, represented by the average $\chi_{\log }^{2}$ value of 0.0014 . Uncertainties occur in size regions where the particle concentration is low. The parameters of the Aitken-mode, $N_{\text {int }}, D_{\text {med }}$, and $\sigma_{\text {geom }}$, are retrieved with average uncertainties of $0.60 \%, 0.39 \%$, and $0.27 \%$, respectively.

\subsection{Condensation particle size analyser and passive cavity aerosol spectrometer probe}

Space and weight are limiting factors for airborne payloads. It is therefore useful to know how much information on the particle size distribution can be extracted from a limited set of instruments. One such configuration is the combination of the CPSA, which is installed inside the aircraft cabin, with a wing-mounted PCASP. ${ }^{5}$ The PCASP is an optical particle counter (OPC) with a sensitive range of $0.1 \mu \mathrm{m}<D_{\mathrm{p}}<3 \mu \mathrm{m}$. For simplicity, the transfer functions of the five smallest size channels of the PCASP are approximated by DMA transfer functions. The shape of the DMA transfer function is similar

${ }^{5}$ Manufacturer: Particle Metrics Inc. (formerly Particle Measuring Systems (PMS)), 5505 Airport Boulevard, Boulder, CO 80301, USA. 

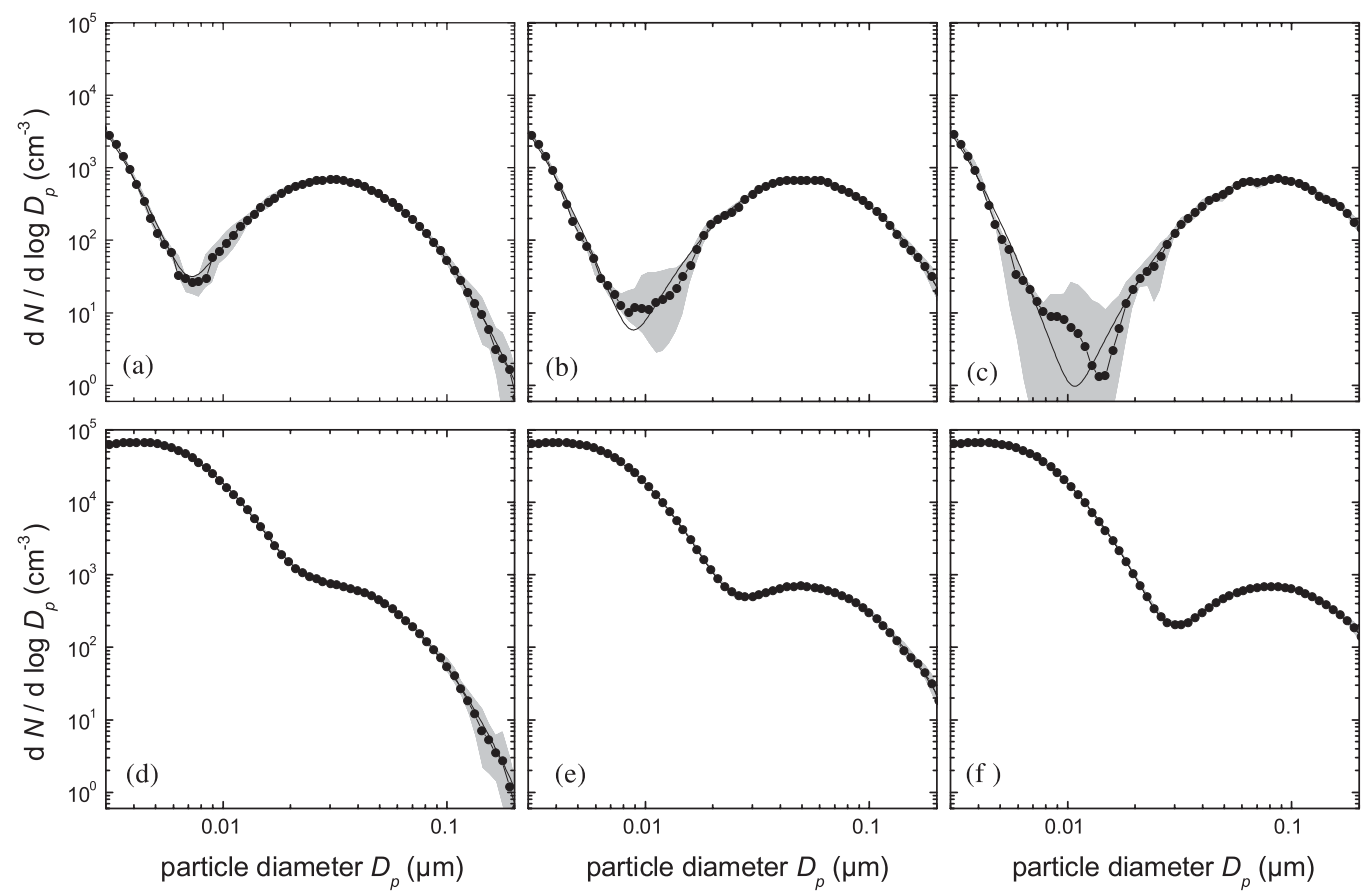

Fig. 4. Same as Fig. 3, but with an assumed Poisson error for the synthetic data. The resulting uncertainty of the particle size distribution was calculated using the Monte-Carlo method (area shaded in grey).

to the shape of the transfer function of one PCASP size channel when particles with more than one elementary charge are removed from the DMA transfer function. The lower sample flow of approximately $1 \mathrm{~cm}^{3} / \mathrm{s}$ in the PCASP as compared to a typical sample flow of $16.66 \mathrm{~cm}^{3} / \mathrm{s}$ in a DMA is taken into account by the low peak sampling efficiency of the DMA in the relevant size range. The peaks of the DMA transfer functions are located at the same particle diameters as the centres of the respective PCASP size channels. Fig. 5 assembles the transfer functions of CPSA and simulated OPC used here to test the performance of this instrument combination. When the PCASP is combined with the CPSA, a window in particle size between $20 \mathrm{~nm}<D_{\mathrm{p}}<0.1 \mu \mathrm{m}$ remains where no information on the particle size distribution is available. It will be investigated whether this information is, nevertheless, sufficient to infer the most important characteristics of the size distribution in the nucleation- and Aitken-mode size range.

As before, the performance test is done by calculating the instrument responses for the six test particle size distributions, using them as input for the inversion algorithm, and compare the inversion output with the test distributions. The result (Fig. 6) still shows a very good agreement between the test size distributions and the inversion results despite the fact that there is no information on the size distribution in the range $20 \mathrm{~nm}<D_{\mathrm{p}}<80 \mathrm{~nm}$ contained in the instrument responses. The agreement is best in the cases (a)-(c) where the size region that is dominated by the Aitken-mode aerosol stretches into the sensitive ranges of both the CPSA and the PCASP. In the remaining cases (d)-(f) where the CPSA sensitive size range is dominated by the nucleation mode and the PCASP sensitive range is dominated by the Aitken- 


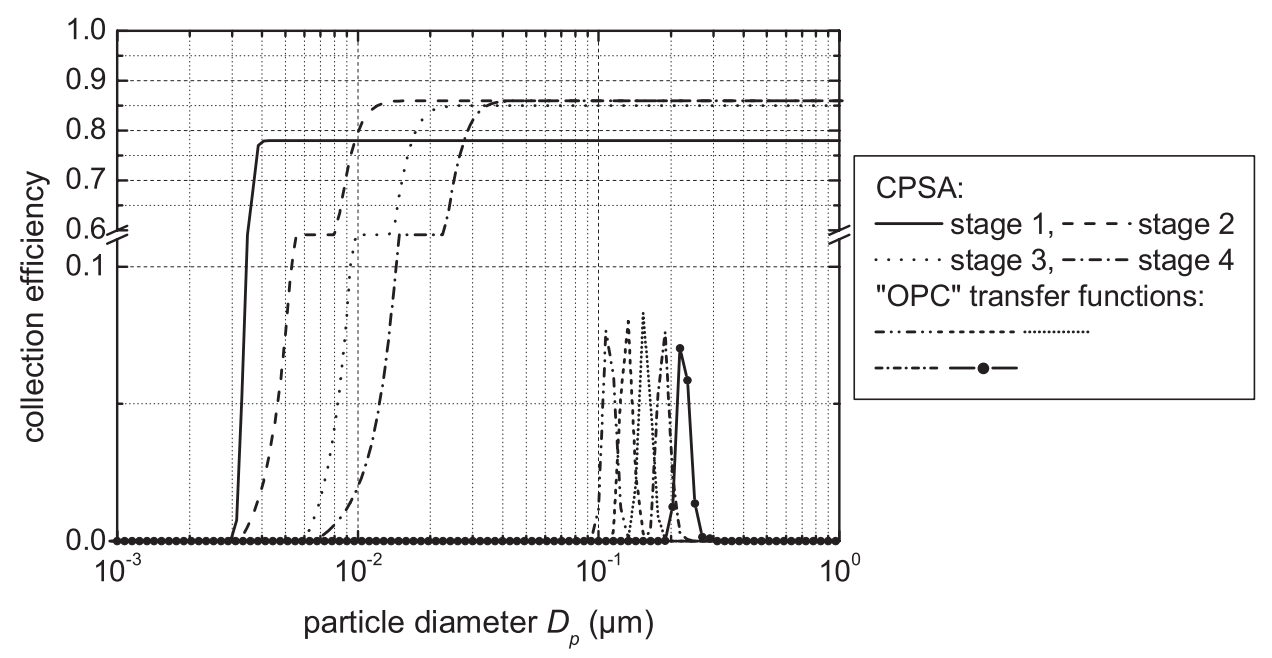

Fig. 5. Transfer functions of the four channels of the CPSA for typical operating conditions, together with transfer functions of the DMA for particle diameters $D_{\mathrm{p}}>100 \mathrm{~nm}$, which are used for simplicity to approximate the transfer functions of an OPC.

mode, the Aitken-mode is not as well reproduced due to lack of information. This is reflected by the average $\chi_{\log }^{2}$ value of 0.0129 which is roughly an order of magnitude larger than that of the CPSA/DMA instrument combination. The parameters of the Aitken-mode are retrieved with $11.9 \%\left(N_{\text {int }}\right), 11.59 \%$ $\left(D_{\text {med }}\right)$, and $4.82 \%\left(\sigma_{\text {geom }}\right)$ uncertainty on average.

\subsection{Condensation particle size analyser and parallel diffusion battery}

A diffusion battery (DB) discriminates particles with different diameters by their diffusion coefficient. A single DB stage consists of a number of fine meshes which are inserted into the aerosol flow perpendicular to the flow direction and a CPC downstream of the meshes that counts the particles penetrating them. Particles are removed by diffusion to, interception on, and impaction onto the fibers. In a common DB, a single $\mathrm{CPC}$ is used and the number of meshes upstream is successively increased and decreased to vary the transfer function. To increase the temporal resolution, a PDB uses several CPCs with different numbers of meshes upstream of their inlets sampling the same aerosol. The three channel PDB used here has a sensitive range of $28 \mathrm{~nm}<D_{\mathrm{p}}<120 \mathrm{~nm}$ with high temporal resolution, complementing the CPSA. The respective transfer functions, which can be calculated from theory (Hinds, 1999), are plotted in Fig. 7a together with those of the CPSA.

Although the instrument combination of CPSA and PDB contains size distribution information in a similar range of $D_{\mathrm{p}}$ as the combination of CPSA and DMA, the data information content is less for the former. The transfer functions of the PDB are much less sharply defined than those of the DMA. To obtain the same amount of information on the size distribution, the uncertainty of PDB data has to be much lower than for DMA data. However, since the DMA has to scan the size range where information on the particle size distribution is to be collected, its temporal resolution is on the order of minutes. In contrast, the temporal resolution of a parallel diffusion battery is on the order of seconds, which makes it particularly suitable for aircraft applications. 

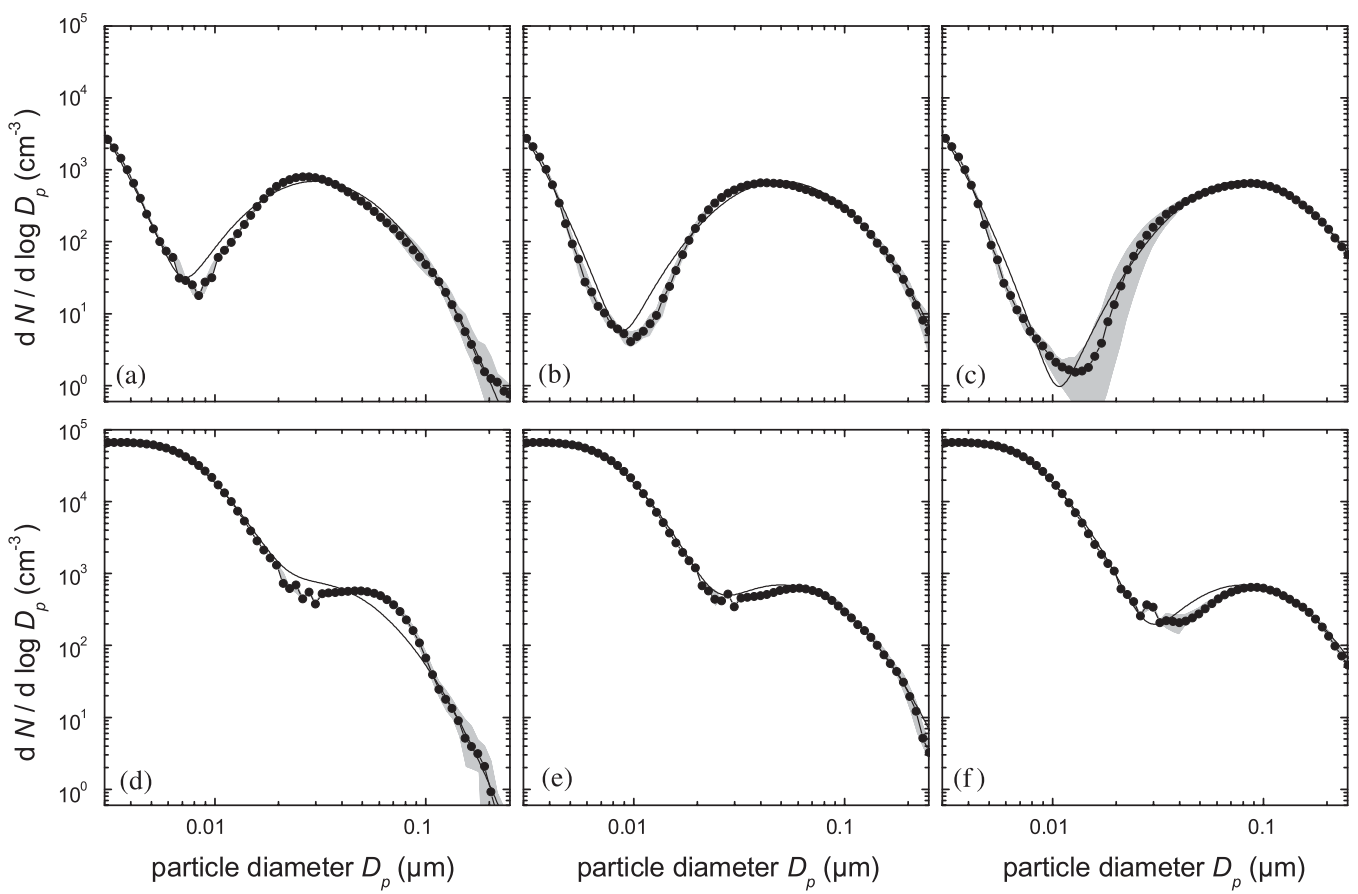

Fig. 6. Comparison of test particle size distributions (Table 1, thin solid line) and particle size distributions inverted using synthetical instrument responses of CPSA and OPC, simulated by a DMA, as input (black dots). The ranges in $D_{\mathrm{p}}$ where CPSA and OPC data contain information on the particle size distribution, $D_{\mathrm{p}}<20 \mathrm{~nm}$ and $D_{\mathrm{p}}>100 \mathrm{~nm}$, respectively, appear in different hatch styles. A Poisson error was assumed for the synthetical data. The resulting uncertainty of the particle size distribution was calculated using the Monte-Carlo method (area shaded in grey).

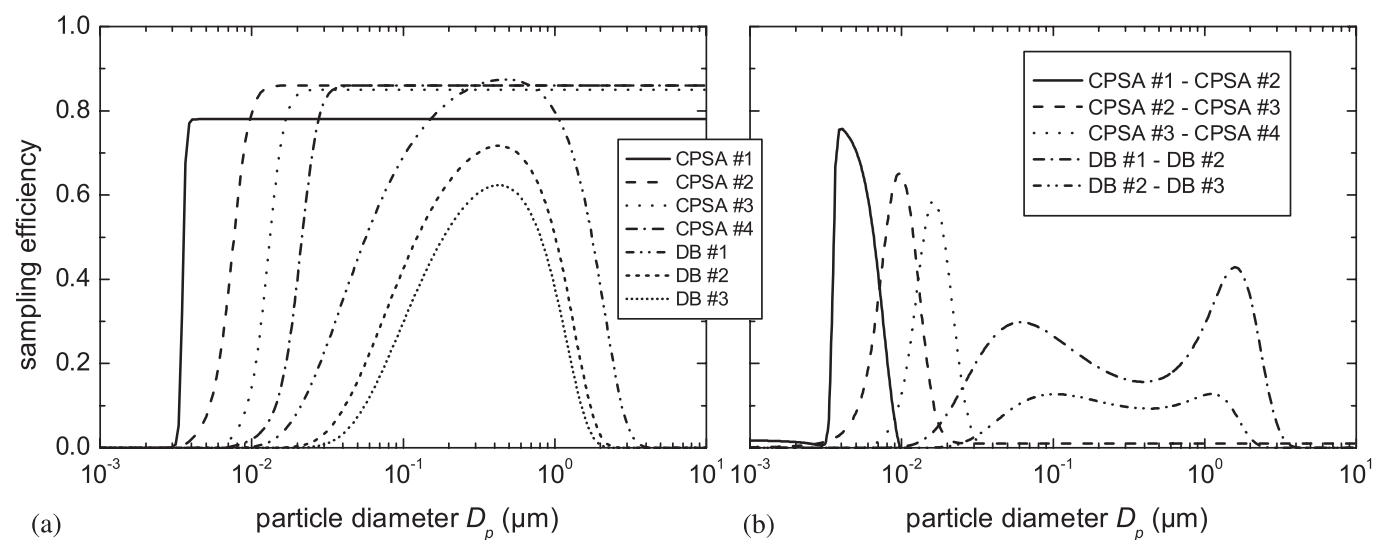

Fig. 7. Transfer functions of the four CPSA channels and three parallel DB stages used in this study. Panel (a) shows the transfer functions of CPSA and PDB, whereas panel (b) plots the difference between the individual CPSA transfer functions and the individual PDB stages. In the difference of the PDB transfer functions, a characteristic double peak structure is visible. 

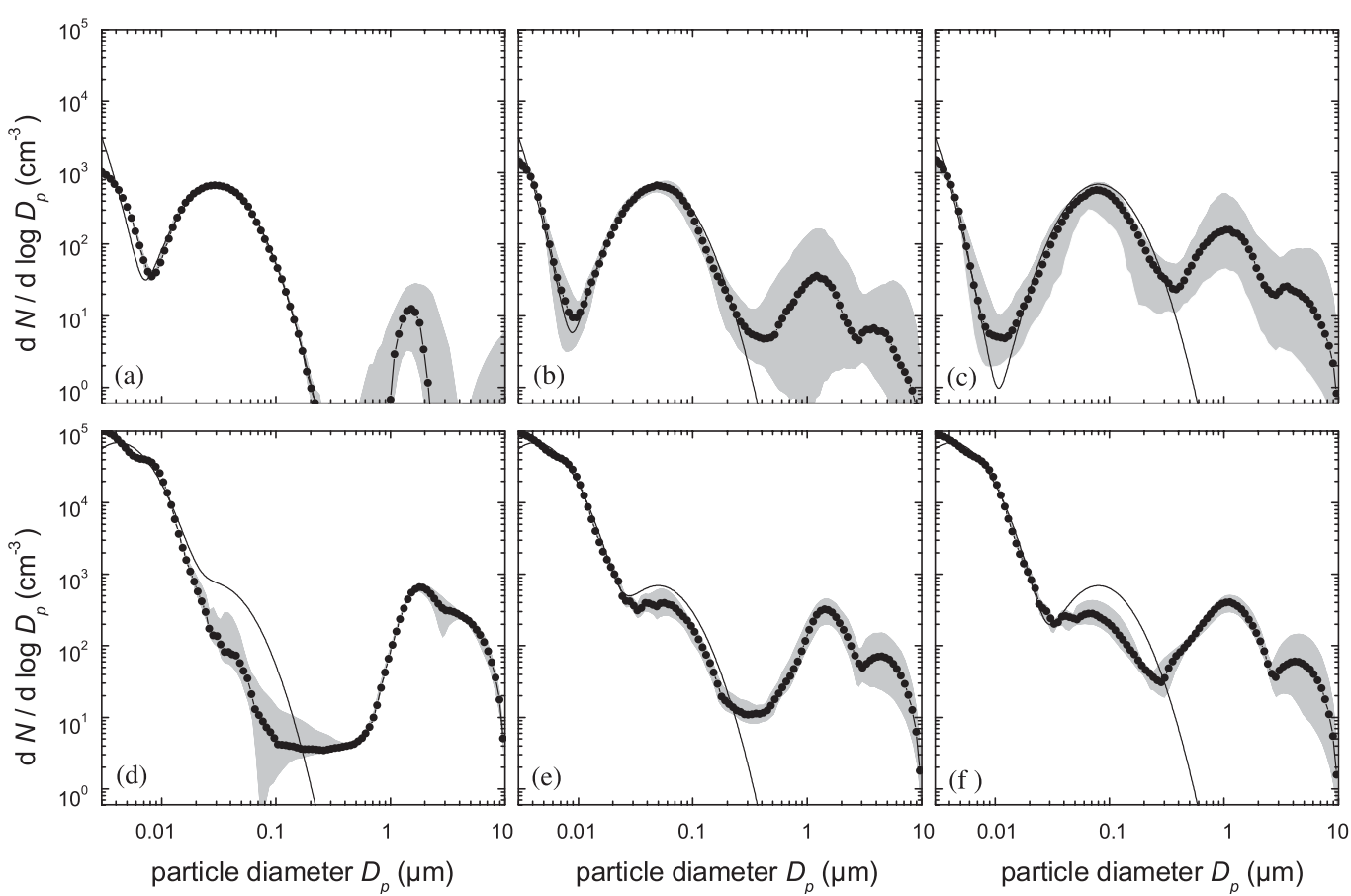

Fig. 8. This Figure compares the test size distributions (Table 1, solid lines) with those obtained by inversion (black dots) of synthetic data of CPSA and a PDB with three stages. The range of particle diameter where the instruments contribute information appears hatched. The uncertainty of the inverted size distribution, resulting from the Poisson error of the synthetic data, is shown in grey.

Another issue that complicates inversion of PDB data is its ambiguity in particle size. The information on the particle size distribution in PDB data is contained in the difference of concentrations measured by different PDB channels. When taking the corresponding difference of the transfer functions of adjacent PDB channels (Fig. 7b), the graph shows two peaks, one in the sub- and one in the supermicron size range. A concentration difference measured by the respective PDB channels can therefore be allocated to two different size ranges.

The effect on the inversion result is investigated in Fig. 8. The ambiguity in the PDB data is visible especially for the test size distributions (d)-(f). In these cases, a significant amount of particles located in the Aitken-mode size range in the test size distributions are aliased into the supermicron size range in the inversion result.

This inherent ambiguity in the PDB data can be eliminated by adding an impactor upstream of the PDB which removes all particles with $D_{\mathrm{p}}>0.3 \mu \mathrm{m}$. Fig. 9a shows the resulting transfer functions of the three PDB channels, Fig. 9b their corresponding differences. It is obvious that the double peak structure that caused the ambiguity in the inversion of PDB data has vanished.

Fig. 10 presents the inversion of the test size distributions using CPSA and the PDB modified with a pre-impactor as described. The test size distributions are now reproduced by the inversion result without aliasing particles from one size range to another. The average $\chi_{\log }^{2}$ value of 0.0174 is comparable in size to the one obtained for the CPSA/PCASP instrument combination. On the other hand, the average 

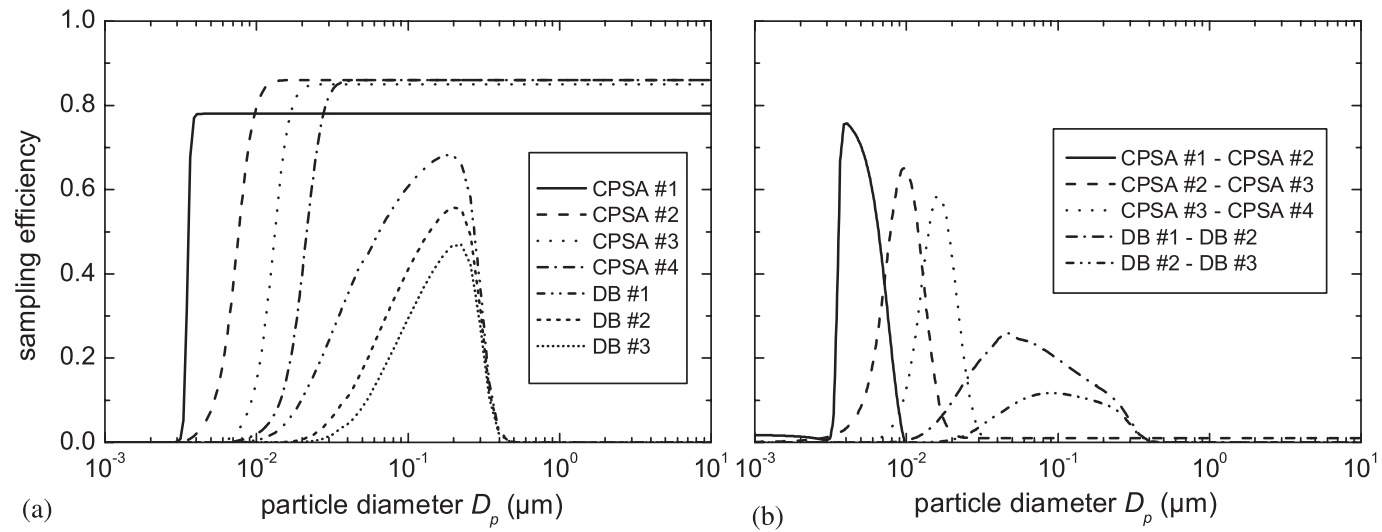

Fig. 9. Transfer functions of the four CPSA channels and three PDB stages used in this study. An impactor with a cut at $0.3 \mu \mathrm{m}$ was added in front of the PDB. Panel (a) shows the modified transfer functions of CPSA and PDB, whereas panel (b) plots the difference between the individual CPSA transfer functions and the individual stages of the modified PDB.
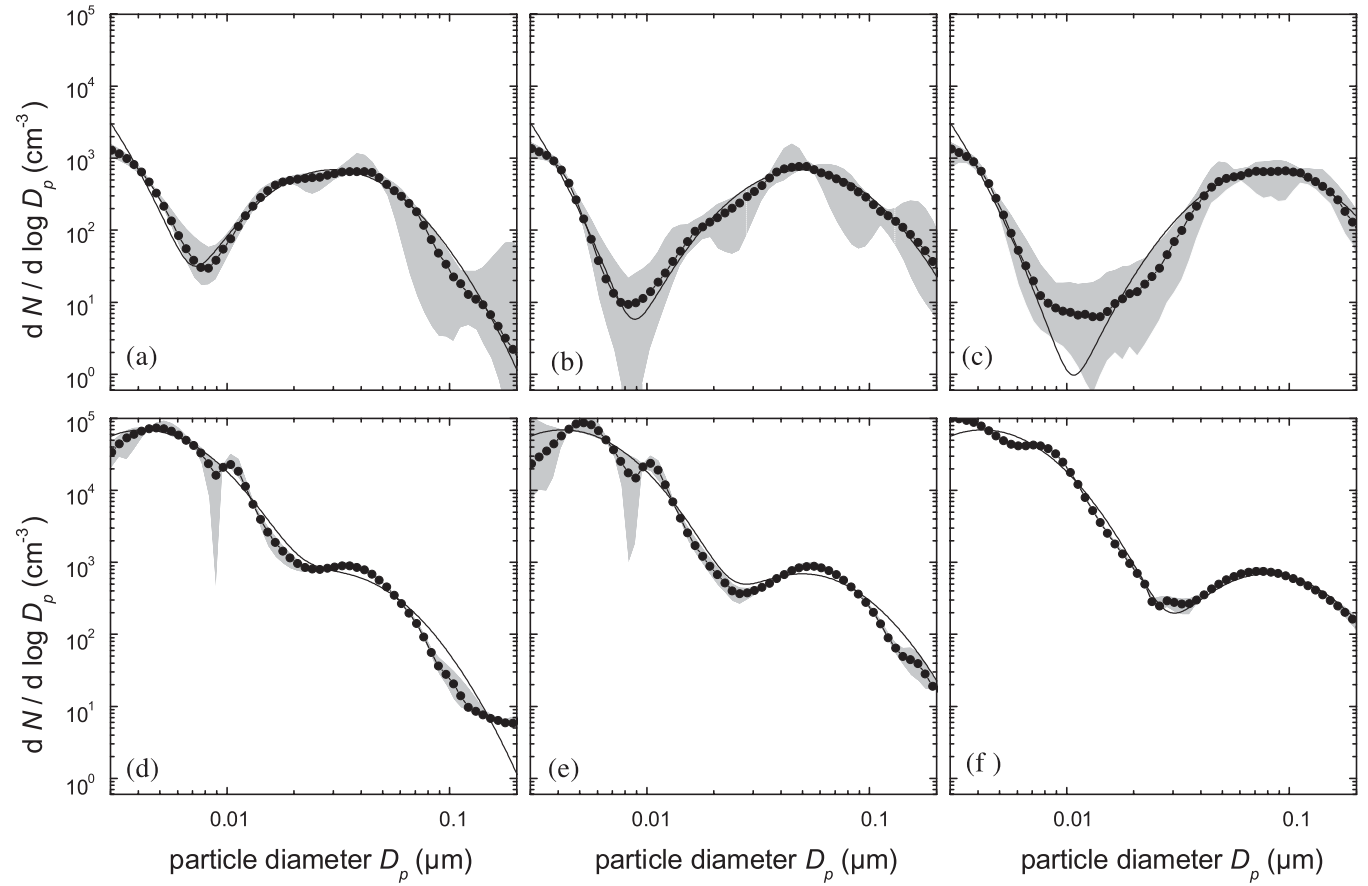

Fig. 10. Comparison of test size distributions (Table 1, solid lines) with respective inversion result based on synthetic data of CPSA and a three stage PDB. The underlying transfer functions of CPSA and PDB are given in Panels (a) and (b) of Fig. 9, i.e. an impactor with $0.3 \mu \mathrm{m}$ cut-off diameter has been installed in the inlet of the PDB. Again, the uncertainty (shaded in grey) was calculated with the Monte-Carlo method assuming a Poisson error of the synthetic data. 
parameter uncertainties of the Aitken-modes retrieved with CPSA and PDB, $4.57 \%\left(N_{\text {int }}\right), 3.61 \%\left(D_{\text {med }}\right)$, and $3.73 \%\left(\sigma_{\text {geom }}\right)$, are significantly lower than for the CPSA/PCASP instrument combination since the latter one does not contain information in the $20 \mathrm{~nm}<D_{\mathrm{p}}<0.1 \mu \mathrm{m}$ range. The size information in PDB data is less well defined than in DMA data. Therefore, the test size distributions are reproduced with higher accuracy by the inversions based on the CPSA/DMA instrument combination than the inversions based on CPSA and PDB data. However, with respect to airborne applications, the CPSA/PDB instrument combination is a valuable alternative to the combination of CPSA and DMA since it offers a higher temporal resolution.

\section{Application to laboratory data}

There are two main sources of uncertainty that make measured data more difficult to invert than calculated instrument responses. On the one hand, the real uncertainty of the data can be larger than predicted using Poisson statistics. Also, the instruments' transfer functions under field conditions may differ from those under lab conditions due to variations in pressure, temperature, and other environment parameters. These variations are usually small but, depending on the particle size distribution, may still be significant and are difficult to characterise. A reliable inversion algorithm should give a stable inversion result even with these sources of uncertainty present.

In this example, measured data obtained with different instrument types on the same aerosol is combined in one inversion, which has so far only been demonstrated in theory (Wolfenbarger \& Seinfeld, 1990). The measurements were done in the laboratory on a soot test aerosol generated by the PALAS ${ }^{6}$ GFG1000 soot aerosol generator. The particle size distribution of the test aerosol was measured in parallel by a TSI $^{7} 3071$ DMA operated in step-scanning mode and by the combination of CPSA and PDB as described in the previous section. One scan of the DMA included 10 logarithmically equidistant steps between $10 \mathrm{~nm}$ and $0.2 \mu \mathrm{m}$ particle diameter. The median diameter of test aerosol was varied by changing the spark frequency of the PALAS GFG-1000 while keeping the gas flow through the spark chamber and the subsequent dilution flow constant. Fig. 11 presents the inversion results for three different spark frequencies of the soot aerosol generator. Due to its higher information content, the DMA inversion result serves as reference measurement for the result of the CPSA/PDB data inversion. The shift in the median diameter of the mono-lognormal particle size distribution with varying spark frequency of the soot generator is visible in both inversion results. In all cases, the particle size distribution retrieved from the CPSA/PDB data agrees with the DMA based result within its uncertainty, despite the steep gradients in the size distributions.

\section{Conclusion}

An algorithm for the inversion of data on aerosol particle size distributions is presented that utilises both the Singular Value Decomposition and the least-squares with equality and inequality constraints algorithms to solve the inversion equation. The algorithm combines the advantages of not needing an

\footnotetext{
${ }^{6}$ PALAS GmbH, Greschbachstr. 3b, D-76229 Karlsruhe, Germany.

7 TSI Inc., 500 Cardigan Road, Shoreview MN 55126, USA.
} 


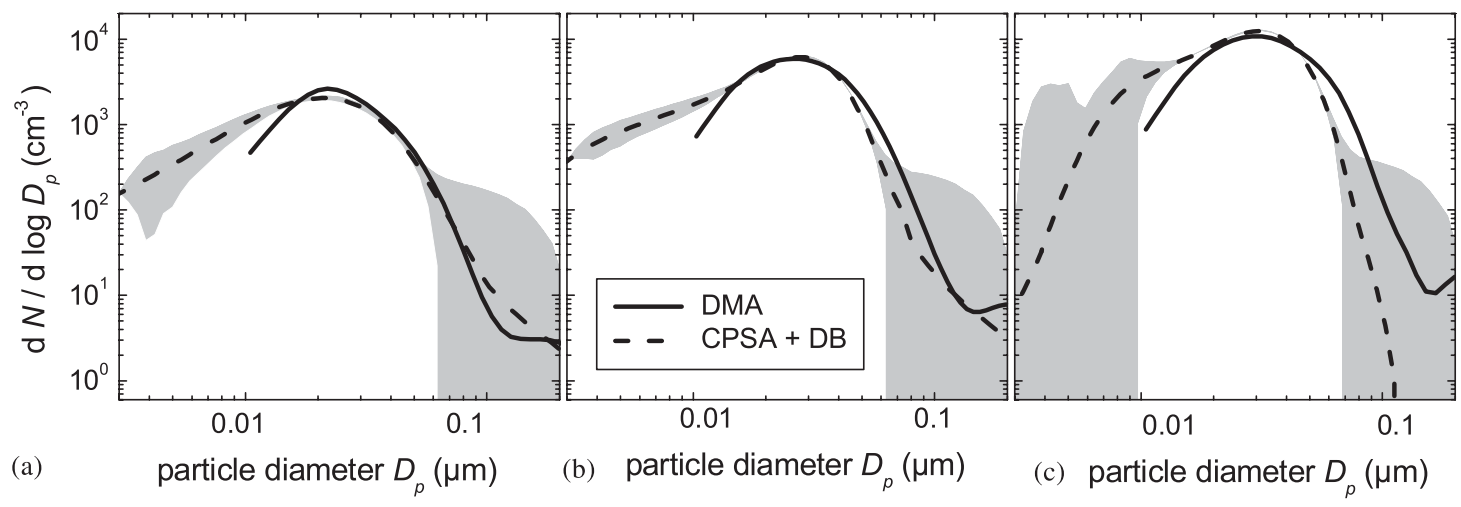

Fig. 11. Comparison of particle size distributions of soot aerosol measured simultaneously by a stepping mode DMA $\left(10 \mathrm{~nm}<D_{\mathrm{p}}<150 \mathrm{~nm}\right)$ (solid line) and by the combination of CPSA and PDB (dashed line). The soot aerosol was produced by the PALAS GFG-1000 aerosol generator. The three panels show particle size distributions for different discharge frequencies of the aerosol generator, resulting in different particle median diameters of the produced soot aerosol. The uncertainty of the inversion result for CPSA plus PDB, based on the Monte-Carlo method and the actual uncertainty of the data, is shaded in grey. The uncertainty of the inversion result for the DMA is given by the line width.

initial guess, reproducing instrument responses accurately, yielding the smoothest non-negative result consistent with the input data, giving the uncertainty of the inversion result based on the uncertainty of the input parameters, covering a dynamic range of more than 4 orders of magnitude in particle concentration, and combining data of different instrument types into one inversion result.

The development of the algorithm has been triggered by the need to interpret data obtained by the CPSA, an instrument designed to measure the particle size distribution of atmospheric aerosol in the size range $3 \mathrm{~nm}<D_{\mathrm{p}}<20 \mathrm{~nm}$ with high temporal resolution using an aircraft platform. Together with instruments yielding information on the particle size distribution for $20 \mathrm{~nm}<D_{\mathrm{p}}$, the performance of the instrument/algorithm combinations has been evaluated using synthetic data. Combining the CPSA with a DMA allows measurement of the particle size distribution for $3 \mathrm{~nm}<D_{\mathrm{p}}<1 \mu \mathrm{m}$ with high size, but moderate temporal resolution. The accuracy of the retrieved particle size distributions was the highest of all instrument combinations tested. If more temporal resolution with the trade-off of reduced size resolution is desired the CPSA may be combined with a PDB. If the instrument package has to be optimised for size and weight, e.g. for aircraft applications, the CPSA may be combined with a PCASP. Both, the CPSA/PDB and the CPSA/PCASP instrument combinations retrieve the particle size distribution in the nucleation and Aitken-mode size range with an accuracy an order of magnitude smaller than the CPSA/DMA instrument combination. However, CPSA with PDB still retrieve the parameters of the Aitken-mode with $\sim 4 \%$ uncertainty whereas the respective value for CPSA plus PCASP is approximately twice as large. The size distributions retrieved from DMA data and CPSA/PDB data obtained simultaneously on the same test aerosol agree within their uncertainties.

The design of the inversion algorithm is general enough to allow for future treatment of instrument data not discussed in this study. For example, the algorithm has also been used to extract the particle size distribution information from data on the spectral particle scattering coefficient (Fiebig \& Ogren, 2004). Besides inversion of size distribution data, the algorithm can also serve as a tool for optimising an instrument's performance depending on the instrument parameters or for designing in- 
strument packages addressing scientific questions that require certain resolutions in time or particle diameter.

\section{Appendix A. Calibration of the CPSA}

The CPSA is optimised for measuring the particle size distribution in the range of $3 \mathrm{~nm}<D_{\mathrm{p}}<20 \mathrm{~nm}$ with a temporal resolution on the order of seconds which is required for deployment on a fast flying jet-engine aircraft.

The four individual channels of the CPSA are similar to the TSI ${ }^{8} 3010$ CPC (Mertes, Schröder, \& Wiedensohler, 1995) but optimised for aircraft use concerning size, weight, and sensitivity to pressure fluctuations in the system. The temperature difference between the CPC's saturation and condensation paths determines the supersaturation reached and therefore the activation threshold diameter of the particles. Since the temperature profile within the condensation path of each CPC is not uniform (Stolzenburg \& McMurry, 1991), the activation threshold diameter is not a sharp limit. By using several CPCs with different threshold diameters in parallel, size distribution information in the nucleation mode range can be obtained with high temporal resolution and statistical significance also at low particle concentrations. The temperature difference in all four CPSA channels can be set to six different pre-chosen values.

\section{A.1. Calibration set-up}

To measure the transfer functions of the four CPSA channels, test aerosols of sodium chloride and ammonium sulphate in the nucleation and Aitken-mode size range were generated by using the tube furnace generator described by Scheibel and Porstendörfer (1983). The tube furnace generator produces particles by evaporation and recondensation of the test substance. After exiting the tube furnace, the generated polydisperse test aerosol was monodispersed with a TSI DMA 3071A (Knutson \& Whitby, 1975) which was operated in a closed-loop set-up. Compromising between width of the particle mode and the particle concentration exiting the DMA, a sheath to sample flow ratio of 10 was used. A TSI 3068 aerosol electrometer served as reference instrument for measuring the counting efficiency of the CPCs as a function of particle diameter. Since particles smaller than $20 \mathrm{~nm}$ are predominantly charged only once (Wiedensohler, 1988), the whole calibration including the measurement of the reference particle concentration can be tracked to first principles. To obtain enough sample for all CPCs and the electrometer, the monodisperse aerosol flow of $1.1 \mathrm{l} / \mathrm{min}$ leaving the DMA was diluted with particle-free air and homogenised in a mixing chamber. Fig. 12 summarises the whole set-up used to calibrate the CPSA.

\section{A.2. Calibration results}

The transfer functions of each CPSA module were calibrated for all six selectable temperature differences $\Delta T$ between saturation and condensation path inside the CPC. The particle concentrations measured by the different instruments were corrected for sampling line losses (Hinds, 1999). Varying $\Delta T$, the transfer function can be varied between 3.5 and $20 \mathrm{~nm}$ particle diameter of $50 \%$ counting efficiency. The position of the ascend in the transfer function moves to larger particle diameters when the temperature

\footnotetext{
${ }^{8}$ TSI Inc., 500 Cardigan Road, Shoreview MN, 55126-3996, USA.
} 

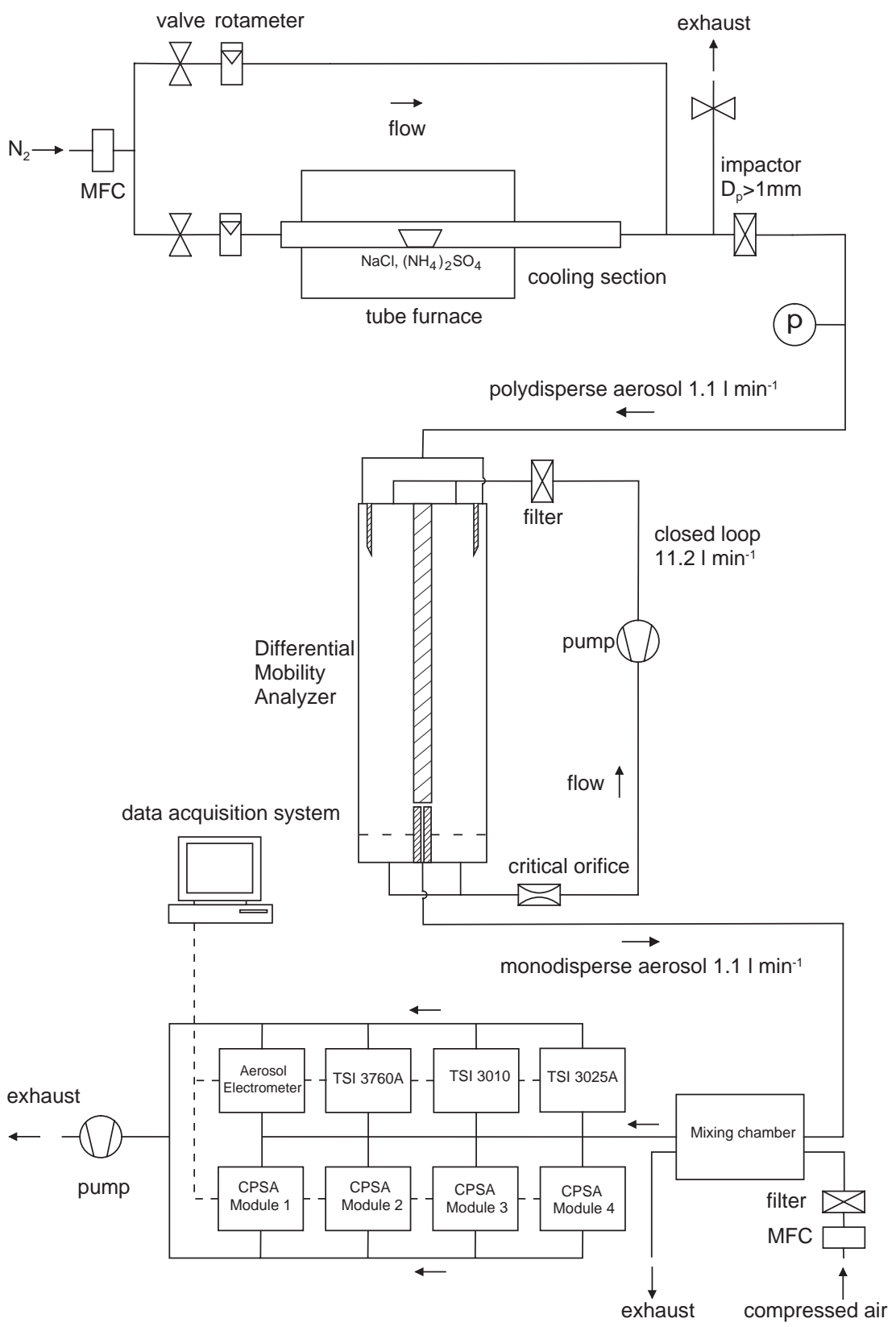

Fig. 12. Experimental set-up for calibrating the four individual CPSA channels, together with other CPCs, and obtaining their transfer functions as a function of supersaturation, i.e. temperature difference between saturater and condenser. 


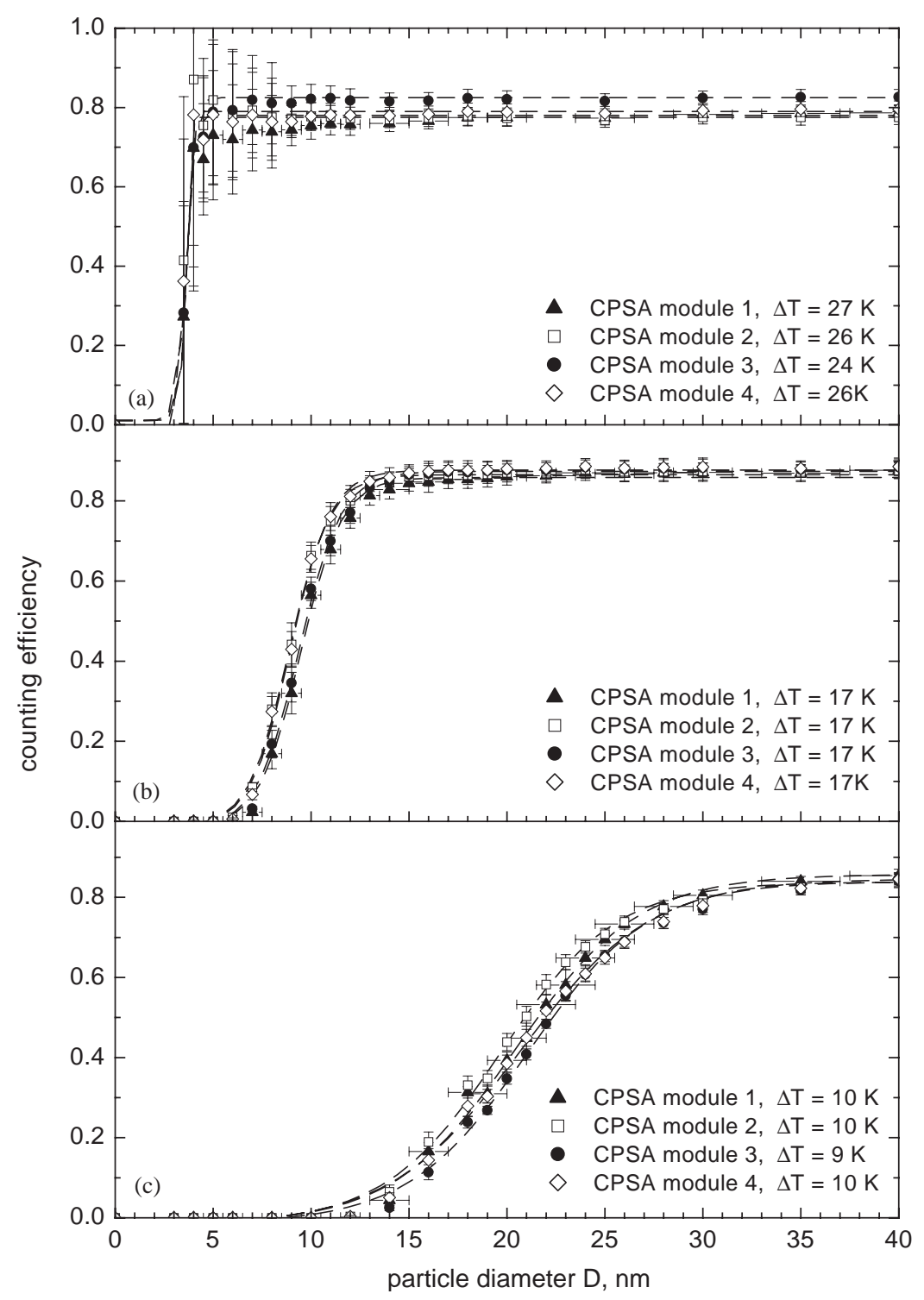

Fig. 13. Transfer functions of all four CPSA modules for three of the six selectable temperature settings.

difference between saturater and condenser decreases, while at the same time the slope of the transfer function becomes smaller. Fig. 13 shows the transfer functions of all four CPSA modules for three selected temperature settings. The transfer functions of different CPSA modules are relatively similar for nominally equal temperature settings, but slight differences exist. To account for these differences, the transfer functions for each CPSA module are stored in look-up tables as a function of the supersaturation reached in the condensation path of the CPC. The internal temperatures of the CPSA modules are measured and recorded together with the measured particle concentration. During inversion, the transfer 
function is interpolated from the look-up tables for each CPSA module and for the exact supersaturation present during the measurement.

\section{References}

Brock, C. A., Schröder, F., Petzold, A., Busen, R., Fiebig, M., Kärcher, B., \& Wilson, J. C. (2000). Particle size distributions measured in the plume of a Boeing 737-300 in flight. Journal of Geophysical Research, 105, 26555-26568.

Fiebig, M., \& Ogren, J. A. (2004). Retrieving aerosol properties by inverse methods of data from several instruments. Fall Meeting of the American Geophysical Union, December 2004, San Francisco.

Haskell, K. H., \& Hanson, R. J. (1981). An algorithm for linear least squares problems with equality and nonnegativity constraints. Mathematical Programming, 21, 98-118.

Hinds, W. C. (1999). Aerosol technology. (2nd ed.), New York: Wiley.

Kandlikar, M., \& Ramachandran, G. (1999). Inverse methods for analysing aerosol spectrometer measurements: A critical review. Journal of Aerosol Science, 30, 413-437.

Knutson, E. O., \& Whitby, K. T. (1975). Aerosol classification by electric mobility: Apparatus, theory and applications. Journal of Aerosol Science, 6, 443-451.

Lekhtmakher, S., \& Shapiro, M. (2000). On the paper: Inverse methods for analysing aerosol spectrometer measurements: A critical review. Journal of Aerosol Science, 31, 867-873.

Mertes, S., Schröder, F., \& Wiedensohler, A. (1995). The particle detection efficiency curve of the TSI-3010 CPC as a function of the temperature difference between saturator and condenser. Aerosol Science and Technology, 23, $257-261$.

O’Dowd, C. D., Hämeri, K., Mäkelä, J. M., Pirjola, L., Kulmala, M., Jennings, S. G., Berresheim, H., Hansson, H.-C., de Leeuw, G., Kunz, G. J., Allen, A. G., Hewiit, C. N., Jackson, A., Viisanen, Y., \& Hoffmann, T. (2002). A dedicated study of new particle formation and fate in the coastal environment (PARFORCE): Overview of objectives and achievements. Journal of Geophysical Research, 107(D19), 8108.

Petzold, A., Fiebig, M., Flentje, H., Keil, A., Leiterer, U., Schröder, F., Stifter, A., Wendisch, M., \& Wendling, P. (2002). Vertical variability of aerosol properties observed at a continental site during the lindenberg aerosol characterization experiment (LACE 98). Journal of Geophysical Research, 107.

Pirjola, L., O’Dowd, C. D., \& Kulmala, M. (2002). A model prediction of the yield of cloud condensation nuclei from coastal nucleation events. Journal of Geophysical Research, 107(D19), 8098.

Press, W. H., Teukolsky, S. A., \& Vetterling, W. T. (1992). Numerical recipes in C. (2nd ed.), Cambridge, New York, Port Melbourne: Cambridge University Press.

Roth, C., Berlauer, U., \& Heyder, J. (1989). Particle size analysis of log-normally distributed ultrafine particles using a differential mobility analyser. Journal of Aerosol Science, 20, 547-556.

Scheibel, H. G., \& Porstendörfer, J. (1983). Generation of monodisperse Ag- and NaCl-aerosols with particle diameters between 2 and $300 \mathrm{~nm}$. Journal of Aerosol Science, 14, 113-126.

Stein, C., Schröder, F., \& Petzold, A. (2001). The condensation particle size analyzer: A new instrument for the measurement of ultrafine aerosol size distributions. Journal of Aerosol Science, 32, S381-S382.

Stolzenburg, M. R., \& McMurry, P. H. (1991). An ultrafine aerosol condensation nucleus counter.Aerosol Science and Technology, $14,48-65$.

Wiedensohler, A. (1988). An approximation of the bipolar charge distribution for particles in the submicron size range. Journal of Aerosol Science, 19, 387-389.

Wolfenbarger, J. K., \& Seinfeld, J. A. (1990). Inversion of aerosol size distribution data. Journal of Aerosol Science, 21, 227-247. 